\title{
Erratum to: Feasibility outcomes of a presurgical randomized controlled trial exploring the impact of caloric restriction and increased physical activity versus a wait-list control on tumor characteristics and circulating biomarkers in men electing prostatectomy for prostate cancer
}

\author{
Wendy Demark-Wahnefried ${ }^{1 *}$, Jeffery W. Nix², Gary R. Hunter ${ }^{3}$, Soroush Rais-Bahrami' ${ }^{2}$, Renee A. Desmond ${ }^{4}$, \\ Balu Chacko ${ }^{5}$, Casey D. Morrow ${ }^{6}$, Maria Azrad ${ }^{1}$, Andrew D. Frugé ${ }^{1}$, Yuko Tsuruta' ${ }^{1}$ Travis Ptacek ${ }^{7}$, Scott A. Tully ${ }^{8}$, \\ Roanne Segal ${ }^{9}$ and William E. Grizzle ${ }^{10}$
}

\section{Erratum}

In this version of this article that was originally published [1] there was an error in the figure "CONSORT diagram (Fig. 3)". The labels of the Experimental (Weight Loss) Intervention and the Wait-List Control are currently incorrect and should be exchanged for one another.

The corrected diagram is shown below:

\section{Author details}

'Department of Nutrition Sciences, University of Alabama at Birmingham (UAB), 346 Webb Nutrition Sciences Bldg., 1675 University Blvd, Birmingham, AL, USA. ${ }^{2}$ Department of Urology, UAB, Birmingham, AL, USA. ${ }^{3}$ Department of Human Studies, UAB, Birmingham, AL, USA. ${ }^{4}$ Department of Preventive Medicine, UAB, Birmingham, AL, USA. ${ }^{5}$ Department of Molecular \& Cellular Pathology, UAB, Birmingham, AL, USA. ${ }^{6}$ Department of Cell, Developmental \& Integrative Biology, UAB, Birmingham, AL, USA. 'Department of Microbiology, UAB, Birmingham, AL, USA. ${ }^{8}$ Urology Centers of Alabama, Birmingham, AL, USA. ${ }^{9}$ Department of Medicine, University of Ottawa, Ottawa, Ontario, Canada. ${ }^{10}$ Department of Pathology, UAB, Birmingham, AL, USA.
Received: 19 December 2016 Accepted: 19 December 2016 Published online: 23 January 2017

\section{Reference}

1. Demark- Wahnefried W, Nix JW, Hunter GR, Rais-Bahrami S, Desmond RA, Chacko B, et al. Feasibility outcomes of a presurgical randomized controlled trial exploring the impact of caloric restriction and increased physical activity versus a wait-list control on tumor characteristics and circulating biomarkers in men electing prostatectomy for prostate cancer. BMC Cancer. 2016;16:61.

* Correspondence: demark@uab.edu

${ }^{1}$ Department of Nutrition Sciences, University of Alabama at Birmingham (UAB), 346 Webb Nutrition Sciences Bldg., 1675 University Blvd, Birmingham, AL, USA 
Demark-Wahnefried et al. BMC Cancer (2017) 17:71

Page 2 of 2

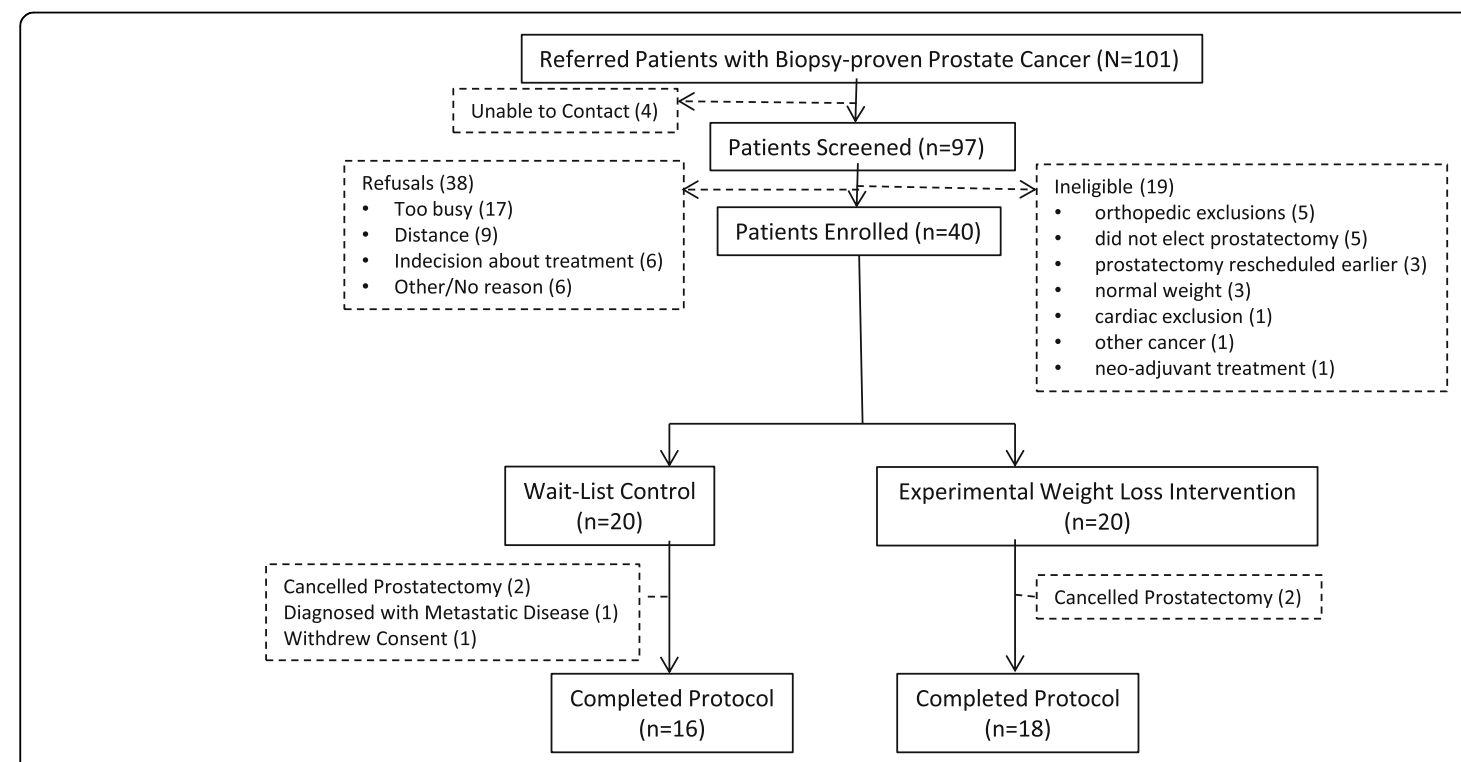

Fig. 3 CONSORT diagram 